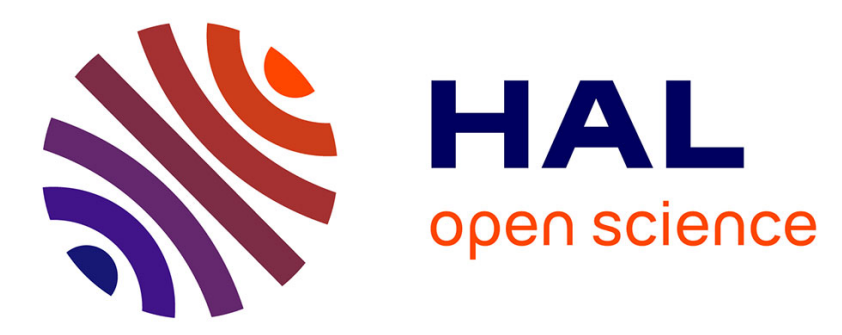

\title{
Physical Mechanisms Inducing Electron Single-Event Upset
}

\author{
Pablo Caron, Christophe Inguimbert, Laurent Artola, Nathalie Chatry, \\ Nicolas Sukhaseum, Robert Ecoffet, Françoise Bezerra
}

\section{- To cite this version:}

Pablo Caron, Christophe Inguimbert, Laurent Artola, Nathalie Chatry, Nicolas Sukhaseum, et al.. Physical Mechanisms Inducing Electron Single-Event Upset. IEEE Transactions on Nuclear Science, 2018, 65 (8), pp.1759-1767. 10.1109/TNS.2018.2819421 . hal-02382999

\section{HAL Id: hal-02382999 \\ https://hal.science/hal-02382999}

Submitted on 2 Nov 2021

HAL is a multi-disciplinary open access archive for the deposit and dissemination of scientific research documents, whether they are published or not. The documents may come from teaching and research institutions in France or abroad, or from public or private research centers.
L'archive ouverte pluridisciplinaire HAL, est destinée au dépôt et à la diffusion de documents scientifiques de niveau recherche, publiés ou non, émanant des établissements d'enseignement et de recherche français ou étrangers, des laboratoires publics ou privés. 


\title{
Physical Mechanisms Inducing Electron Single-Event Upset
}

\author{
P. Caron ${ }^{\circledR}$, Student Member, IEEE, C. Inguimbert, L. Artola ${ }^{\circledR}$, Member, IEEE, N. Chatry, \\ N. Sukhaseum, R. Ecoffet, Member, IEEE, and F. Bezerra
}

\begin{abstract}
With the increase of sensitivity of devices to singleevent upsets (SEUs), the possibility to trigger an upset with incident electrons has been recently raised. All the mechanisms susceptible to trigger the SEUs are investigated in detail. New measurements performed on the field programmable gate array static random access memory based from Xilinx Spartan 6 at $1 \mathrm{MeV}$ seem to confirm two SEU regions with the transition located around $10 \mathrm{MeV}$.
\end{abstract}

Index Terms-Electron, field programmable gate array (FPGA) static random access memory (SRAM) based, SEE, single-event upset (SEU).

\section{INTRODUCTION}

$\mathbf{I}$ N RECENT years, some studies have shown the increase in the sensitivity of memory devices to light particles such as electrons [1]-[6]. Indeed, both dimensional and supply voltage reduction tend to make these devices more and more vulnerable to single-event effects. Different physical mechanisms can be at the origin of these phenomena: direct ionization [1], [4], elastic Coulomb scattering [3], and inelastic nuclear scattering [5].

Inguimbert et al. [3] have highlighted the relatively important contribution of the Coulomb scattering process: at low energy (up to about $10 \mathrm{MeV}$ ), direct ionization seems to be the main process, whereas at higher energies, Coulomb scattering seems to dominate. The last point is also validated in [7].

This is quite different from what is observed for heavier particles for which the dominant processes are the ionization and the nuclear inelastic interactions. For heavy ions that have big LETs, the direct ionization is the main contributor to the single-event upset (SEU) rate. At high energy (>10 MeV), protons produce mainly indirect SEUs by means of nuclear interactions. Low-energy protons $(\sim 10 \mathrm{MeV})$ that have a higher LET are also demonstrated to produce SEU by direction ionization.

The experimental measurements performed by Samaras et al. [2] on the field programmable gate array (FPGA) Xilinx Spartan 6 show clearly, around $10 \mathrm{MeV}$,

P. Caron, C. Inguimbert, and L. Artola are with DPhIEE, ONERA, 31055 Toulouse, France (e-mail: pablo.caron@onera.fr; christophe.inguimbert@onera.fr; laurent.artola@onera.fr).

N. Chatry and N. Sukhaseum are with TRAD, 31670 Labège, France (e-mail: nathalie.chatry@trad.fr; nicolas.sukhaseum@trad.fr).

R. Ecoffet and F. Bezerra are with CNES, 31401 Toulouse, France (e-mail: robert.ecoffet@cnes.fr; francoise.bezerra@cnes.fr). an increase of the sensitivity correlated with the electronuclear reaction threshold. And even if this increase has been shown to be correlated with the elastic Coulomb scattering process [3], the importance of the electronuclear reaction process should be investigated.

Indeed the electron/Si elastic scattering process is able to produce silicon recoils having energies greater than some kiloelectronvolts, which corresponds to the critical energies of integrated devices studied here. Similarly, electronuclear reactions are able to produce energetic recoil elements that able to trigger upsets.

This paper has two main goals: first, validate the presence of an SEU threshold around $10 \mathrm{MeV}$ by performing some tests at lower energy $(1 \mathrm{MeV})$ and second, identify all the physical mechanisms responsible for this behavior.

A focus will be made on the electronuclear process. The importance of the Bremsstrahlung effect will also be investigated. Indeed at high energy ( $>10 \mathrm{MeV})$, electrons produce during their slowdown a large amount of energetic gamma photons (Bremsstrahlung) that are able themselves to initiate other processes like photonuclear reactions. The contribution of these mechanisms shall also be investigated.

In Section I, this paper describes the different physical processes potentially responsible for the SEUs:

1) inelastic electron/electron process (direct ionization);

2) elastic Coulomb electron/Si scattering process;

3) electronuclear reactions;

4) processes induced by Bremsstrahlung photons.

The nature and the energy of the products of these reactions will be described. Then, by means of a very simple rectangular parallelepipedic (RPP) approach, the relative importance of these different mechanisms on the SEU sensitivity will be evaluated. These calculations have been performed using the GEANT4 (v10.2) C++ library [8]-[10] that allows the transport of electrons at high energy including a model of electronuclear processes.

A comparison has been performed with experimental measurements made on the Xilinx Spartan 6. The data of Samaras et al. [2] have been complemented with new measurements made at $1 \mathrm{MeV}$.

\section{Physical Mechanisms}

\section{A. Direct Ionization}

First, electrons can produce inelastic interactions with electrons of the target material (in this paper, silicon). The produced secondary electrons can in turn interact with other 


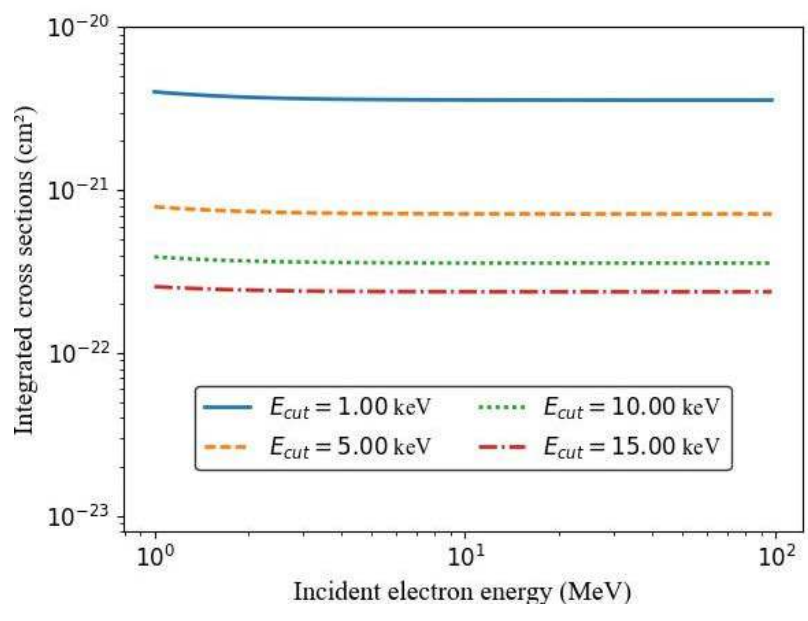

Fig. 1. Integrated Moller cross sections for four energy cuts 1, 5, 10, and $15 \mathrm{keV}$.

TABLE I

Mean Free Path of Ionization as a Function of Incident ELECTRON ENERGY

\begin{tabular}{|c|c|c|c|c|c|}
\hline $\begin{array}{c}\text { Energy } \\
(\mathrm{MeV})\end{array}$ & 0.01 & 0.1 & 1 & 10 & 100 \\
\hline$\lambda(\mathrm{nm})$ & 16.3 & 100.6 & 241.6 & 205.5 & 160.2 \\
\hline
\end{tabular}

electrons and form an ionization shower. If this cascade of interactions is induced in a sensitive region of a memory point and if the deposited energy is large enough, an SEU can be induced. Unlike heavy particles that produce a continuous high level of ionization along their paths, energetic electrons have a very low ionization power. As shown in Table I, the mean free path of the electrons of several megaelectrons in silicon volume is relatively large [11].

Electrons of a few kiloelectronvolts have a mean free path of the same order of magnitude as the sensitive regions of devices of the latest technologies. However, for higher energies, electrons are likely to cross the volume without interacting. Fig. 1 presents the integrated Moller cross section [10] for different energy cuts. It represents the probability to get a secondary electron of energy greater than the cut. One can notice the nearly constant cross section within the energy range of interest [1-100] MeV. Thus, a roughly constant SEU cross section is expected by means of this mechanism.

\section{B. Elastic Coulomb Scattering}

Second, electrons can interact with nuclei by means of elastic Coulombic interactions. This scattering process can remove target nuclei from their site in the lattice.

These recoil ions are highly ionizing particles and they are able to deposit enough charge in the sensitive region of the devices to trigger some SEUs. The maximum energy that can be transferred during this interaction is given by the following equation:

$$
Q_{\max }=\frac{2 M\left(\gamma^{2}-1\right) m^{2}}{m^{2}+M^{2}+2 \gamma m M} .
$$

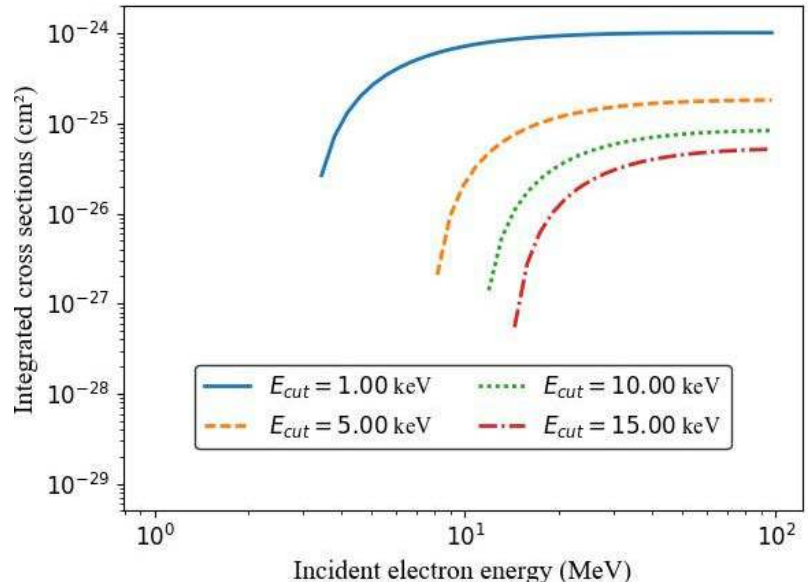

Fig. 2. Integrated Mott cross sections [12] for four energy cuts 1, 5, 10, and $15 \mathrm{keV}$. Exponential charge distribution $F(q)$ is used.

TABLE II

$Q_{\text {max }}$ OF $\mathrm{e}^{-}+$Si Elastic Coulomb SCATTERING AS A FunCtion of INCIDENT ELECTRON ENERGY

\begin{tabular}{|c|c|c|c|c|c|}
\hline $\begin{array}{c}\text { Energy } \\
(\mathrm{MeV})\end{array}$ & 0.1 & 1 & 10 & 100 & 1000 \\
\hline $\begin{array}{c}Q_{\max } \\
(\mathrm{keV})\end{array}$ & $8.58 \cdot 10^{-3}$ & 0.15 & 8.42 & 766.41 & $7.11 \cdot 10^{4}$ \\
\hline
\end{tabular}

With $m$ and $M$ being, respectively, the electron mass $(0.511 \mathrm{MeV})$ and the target nucleus mass and $\gamma$ being the Lorentz factor. Specific notation will be used in the following of this paper: $Q$ will be attributed to recoil energy, $k$ to gamma energy, and $T$ to electron energy. Table II shows the values of $Q_{\max }$ for different incident energies in the case of $\mathrm{e}^{-}+\mathrm{Si}$ reaction.

In very large-scale integrated technologies, the threshold energies for triggering an SEU are of the order of some kiloelectronvolts [3]. Therefore, the incident electron energy must be of the order of $10 \mathrm{MeV}$ in order to generate a recoil nucleus $Q>\sim \mathrm{keV}$. This value is comparable to the threshold SEU cross section reported by Samaras et al. [2]. The screened differential cross section accounting for the finite nuclear size effects is given by Boschini et al. [12]. Fig. 2 presents the integrated Mott relation [12] for different energy cutoffs. At first order, the SEU cross section is expected to be proportional to that cross section. The correlation with the measured SEU threshold ( $\sim 10 \mathrm{MeV}[2])$ is obvious.

\section{Electronuclear Reaction}

Third, electrons are able to trigger some nuclear reactions. The so-called electronuclear process has a threshold around some tens of megaelectronvolt and is able to produce some energetic recoil elements with enough energy to trigger some upsets.

The electronuclear reactions are closely connected with the photonuclear process. Within the equivalent photon approximation, the flux of energetic photons can be folded with the photonuclear cross sections to estimate the electronuclear cross sections. The nuclear reactions induced by energetic photons 


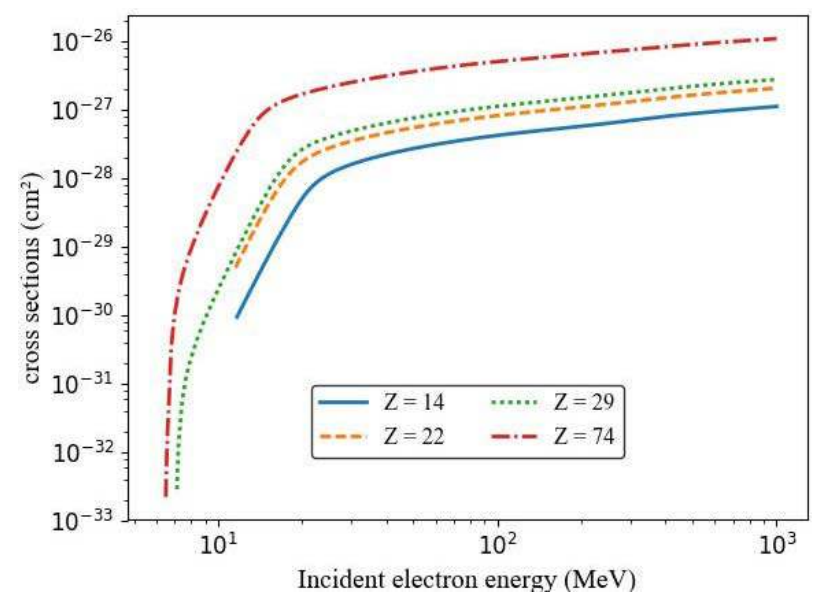

Fig. 3. Total cross sections of electronuclear reaction for silicon $(Z=14)$, titanium $(Z=22)$, copper $(Z=29)$, and tungsten $(Z=74)$ target materials.

$(>10 \mathrm{MeV}$ ) have been widely studied by the nuclear physics community and some cross-sectional databases exist [13], [14] for many materials. In this paper, the GEANT4 [8], [9] toolkit has been used to estimate the electronuclear cross sections. The GEANT4 photonuclear and electronuclear databases were established using the different methods described in [15] and [16] which include the proposed parametric models. To simulate final states for electronuclear reactions, GEANT4 uses the Bertini cascade [17] at low energies and the FRITIOF precompound model [18] at high energies, where the photon is converted to $\pi^{0}$. In the energy range studied in this paper, the Bertini cascade is applied. GEANT4 has also been used to study the nature and the energy distributions of the recoil nuclei. This study has been performed over a wide energy range that goes from some megaelectronvolts up to some gigaelectronvolts. Fig. 3 shows some total electronuclear cross sections calculated with GEANT4 for different target materials $(Z=14,22,29$, and 74). As can be seen in Fig. 3, in the case of silicon, the electronuclear cross section reaches the millibarn level for incident energies of some gigaelectronvolts.

Unlike Rutherford scattering that produces a single recoil ion, electronuclear interactions produce different recoil nuclei simultaneously. A combination of heavy and light elements is produced with energies up to some megaelectronvolts.

Our GEANT4 application was used to analyze the characteristics of the various recoil nuclei produced by electronuclear reactions. The detail of this analysis is presented further in this paper.

\section{Bremsstrahlung-Induced Processes}

The effect of the Bremsstrahlung processes has been simulated thanks to the Seltzer and Berger model [19], where the bremsstrahlung differential cross sections have been tabulated for a wide range of energies and various materials. The SEU yield of the Bremsstrahlung photon spectrum can be evaluated thanks to the sum of the contribution of each energy of the secondary photon distribution

$$
\sigma_{\mathrm{BI}}(E)=\int_{E_{\mathrm{cut}}}^{T} \frac{d \sigma_{B}}{d k} \eta d x \sigma_{I}(k) d k
$$

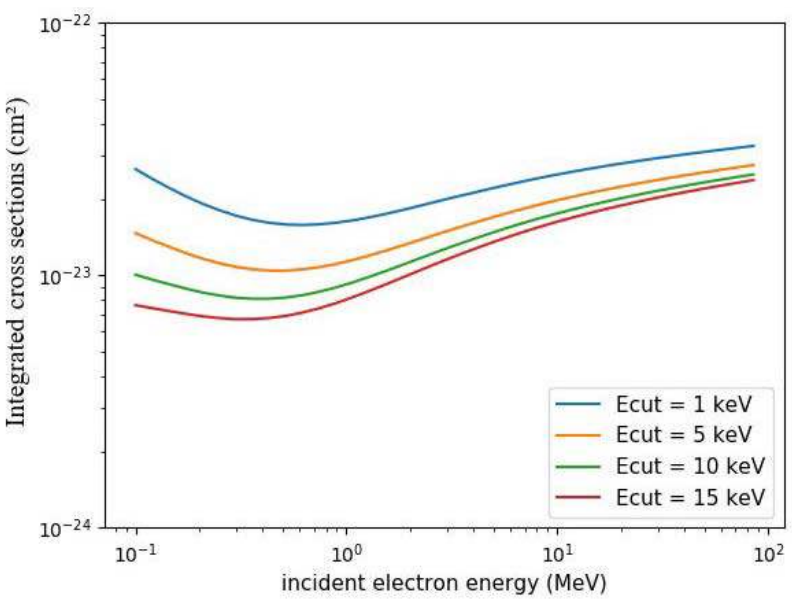

Fig. 4. Integrated cross sections of Bremsstrahlung process derived by Seltzer and Berger data. Different cutoffs are applied: 1, 5, 10, and $15 \mathrm{keV}$.

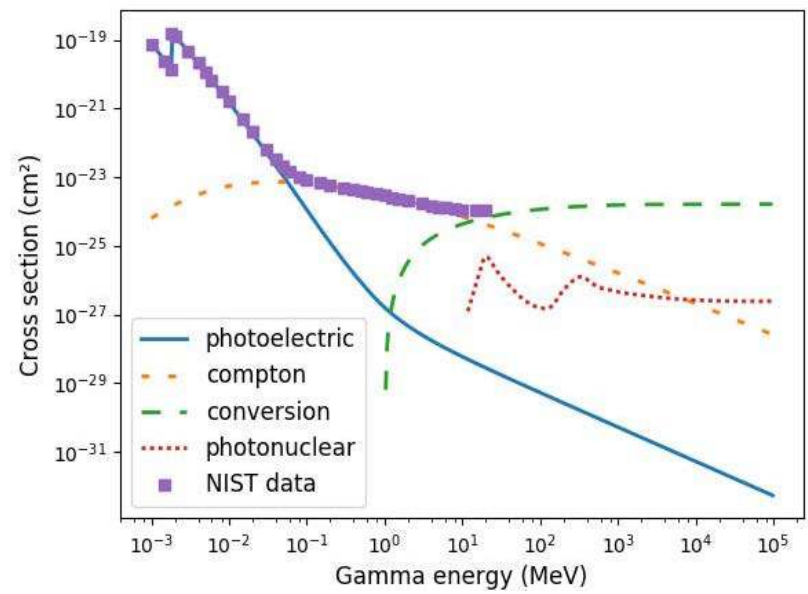

Fig. 5. Cross section of different interactions induced by photons and producing secondary electron or heaviest elements in silicon target. NIST data [20] provide the sum of all interaction cross sections.

With $\sigma_{I}$ being the total cross sections of the process $I$ for a photon having an energy $k$ and $\left(d \sigma_{B} / d k\right) \eta d x$ being the bremsstrahlung spectrum produced by a target of thickness $d x$ and atomic density $\eta\left(\eta_{\mathrm{Si}} \sim 5 \times 10^{22} \mathrm{~cm}^{-3}\right) . T$ is the incident electron energy and $E_{\text {cut }}$ is the cutoff energy determined according to process $I$. Equation (2) overestimates the bremsstrahlung-induced contributions since it does not take into account the path of the photons generated before the interaction. However, it offers us a simple way to get an idea of the orders of magnitude.

Fig. 4 presents the bremsstrahlung integrated differential cross sections using the Seltzer and Berger model. We note that this process is more probable (some tens of barns) to occur than elastic Coulombic ( $\sim$ tens of millibarn) and electronuclear processes $(<$ millibarn).

Secondary photons can produce nuclear reactions, but also secondary electrons (Compton, photoelectric and pair productions, also called conversion) that can trigger some SEUs. To have an idea of the importance of these processes, we have used different models implemented in GEANT4 [10] (Fig. 5). 


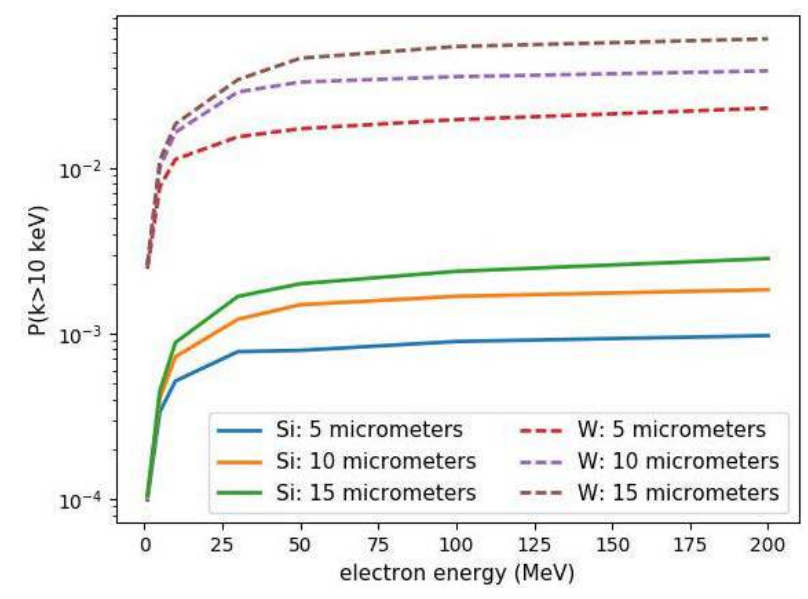

Fig. 6. Probability to have a photon with energy $k>10 \mathrm{keV}$ (and induced by bremsstrahlung process) that crosses through the sensitive volume $(500 \times$ $500 \times 500 \mathrm{~nm}^{3}$ ). Two cases are studied: layer of tungsten (W) and layer of silicon ( $\mathrm{Si}$ ) and three dimensions are studied: 5, 10, and $15 \mu \mathrm{m}$.

A simple analysis allows us to evaluate the importance of bremsstrahlung-induced processes in SEU rate calculations. Consider a sensitive volume of $500 \times 500 \times 500 \mathrm{~nm}^{3}$ collapsed under a larger volume, corresponding of the overlayers. The electron gun is positioned at the top center of this geometry. Fig. 6 relates the probability that a photon of more than $10 \mathrm{keV}$, generated by bremsstrahlung, crosses through the sensitive volume. We consider two cases where the overlayers are composed of silicon or tungsten, and we play with geometry dimension. We deactivated the photon-matter interactions so that the results obtained overestimate reality. To describe overlayers, Gadlage et al. [5] used $\mathrm{SiO}_{2}$ and aluminum layers. We therefore treat a worst case (unrealistic) by considering a tungsten volume.

Consider the worst case: $15 \mu \mathrm{m}$ of tungsten and all the photons generated interaction by photoelectric effect with the highest associated cross section, $\sigma_{\text {phot }}(k=10 \mathrm{keV}) \sim$ $10^{-21} \mathrm{~cm}^{2}$. We obtain $10^{-21} \times 5 \times 10^{-2}=5 \times 10^{-23} \mathrm{~cm}^{2}$. This estimation can be directly compared with the integrated differential cross section of the direct ionization process: $\sigma_{\text {ioni }}\left(E_{\text {cut }}=10 \mathrm{keV}\right) \sim 5 \times 10^{-22} \mathrm{~cm}^{2}$. So, despite our assumptions that strongly overestimate our analysis, bremsstrahlunginduced SEU contribution is negligible. This conclusion does not take into account the shielding [21] and also assumes a critical energy of about $10 \mathrm{keV}$ (the order of magnitude of the critical energies that we used in the following of this paper).

\section{SEU SENSITIVITY}

\section{A. Monte Carlo Toolkit}

The GEANT4 library (v10.2) [8], [9] has been chosen to simulate the transport of the incident electron and secondary particles generated in nanometric volumes. GEANT4 is a $\mathrm{C}++$ toolkit assembled by an international collaboration for describing radiation interaction with matter. We have developed some applications to investigate the problematics presented in this paper. The calculations of the energy deposited by incident electrons, in small volumes having dimensions
TABLE III

CHOSEN PARAMETERS FOR THE SRAM/FPGA

\begin{tabular}{|c|c|}
\hline Number of bits (BRAM) & $131072 \times 4 \mathrm{~b}$ \\
\hline Qc & {$[0.30-0.60] \mathrm{fC}$} \\
\hline Sensitive thickness & $50-500 \mathrm{~nm}$ \\
\hline Sensitive area & $0.04-0.4 \mathrm{um}^{2}$ \\
\hline
\end{tabular}

representative of the sensitive volumes of devices of latest generation, have been performed. The MicroElec module of GEANT4 [22] has been chosen to model the transport of electrons down to some electronvolts. The interest of this model is that electrons are generated and transported down to $16.7 \mathrm{eV}$ allowing creation (almost) in its entirely of the generated cascade.

We have developed two applications. The first one samples the population of recoil ions of electronuclear and photonuclear reactions (nature, direction, energy,...). A database of the characteristics of the recoil ions has been established for several material targets. The second application simulates the energy deposited by the recoil particles in a sensitive volume. This task can be performed for nuclear recoil coming from nuclear reactions and secondary electrons produced by means of inelastic electron/electron interactions.

\section{B. RPP Approximation}

The SEU rate is simply calculated according to the RPP approximation [3], [23]-[25].

An SEU will occur only if the deposited energy is greater than a critical value $\left(E_{c}\right)$, corresponding to a critical charge $\left(Q_{c}\right)$. In silicon, the average energy required to generate an electron-hole pair is $3.6 \mathrm{eV}$ [26]. The deposited energy is converted to a critical charge thanks to the conversion factor $22.5 \mathrm{MeV} / \mathrm{pC}$. All the parameters used during the simulations are listed in Table III and are representative of the static random access memory (SRAM)/FPGA under test (Spartan 6 XC6SLX9). These parameters are included between those used by Samaras et al. [2], Inguimbert et al. [3], and Gadlage et al. [5].

Despite the intrinsic limitations of the RPP approximation given the level of integration studied here, this approach is still used by King et al. [1], Samaras et al. [2], Inguimbert et al. [3], Trippe et al. [4], and Gadlage et al. [5]. Our objective being to evaluate the relative contribution of different involved processes; RPP approximation seems relevant considering obtained results.

\section{SEU Cross-Sectional Calculations}

At the first order, the SEU cross sections for the device under test (DUT) can be expressed according to the following equation:

$$
\sigma_{\mathrm{SEU}}=\eta \times S \times h \times N_{\mathrm{bits}} \times \sigma_{I} \times P\left(E_{\mathrm{dep}}>E_{c}\right)
$$


TABLE IV

Mean Recoil Kinetic Energy in Megaelectronvolts During an Electronuclear (Bottom Left) and Photonuclear (Top Right) Reactions as a Function of Incident Particle Energy. Four Groups are Distinguished: Protons, Neutrons, Light Nuclei $(Z<6)$, AND HEAVY NUCLEI $(Z>=6)$

\begin{tabular}{|c|c|c|c|c|c|c|c|c|}
\hline $\begin{array}{l}\text { gamma } \\
\text { electron }\end{array}$ & $12 \mathrm{MeV}$ & $15 \mathrm{MeV}$ & $20 \mathrm{MeV}$ & $50 \mathrm{MeV}$ & $100 \mathrm{MeV}$ & $300 \mathrm{MeV}$ & $500 \mathrm{MeV}$ & $1000 \mathrm{MeV}$ \\
\hline Proton & $0.000 \quad 0.000$ & $2.145 \quad 2.346$ & $3.231 \quad 3.785$ & $4.199 \quad 6.680$ & 4.525 & 10.558 & 18.289 & $27.239 \quad 112.502$ \\
\hline Neutron & $\begin{array}{ll}0.718 & 1.039 \\
\end{array}$ & 1.435 & $2.217 \quad 2.189$ & $2.898 \quad 4.129$ & 3.842 & $\begin{array}{l}38.992 \\
16.400\end{array}$ & 29.047 & $\begin{array}{ll}43.582 & 125.442 \\
\end{array}$ \\
\hline Light nuclei & $0.000 \quad 0.000$ & $0.000 \quad 0.000$ & $\begin{array}{l}5.736 \\
5.182\end{array}$ & $\begin{array}{rr}7.277 & 9.268 \\
\end{array}$ & $\begin{array}{r}7.349 \\
\quad 8.927 \\
\end{array}$ & 7.765 & $8.389 \quad 9.787$ & 8.550 \\
\hline Heavy nuclei & 0.005 & $\begin{array}{ll} & 0.066 \\
0.019 & \\
\end{array}$ & $0.106 \quad 0.186$ & $\begin{array}{ll} & 0.570 \\
0.220 & \end{array}$ & $\begin{array}{ll} & 1.146 \\
0.247 & \end{array}$ & $0.486 \quad 2.664$ & $0.734 \quad 3.048$ & $0.946 \quad 3.231$ \\
\hline \multicolumn{9}{|c|}{ Details of heavy nuclei } \\
\hline Silicon & $\begin{array}{ll} & 0.007 \\
0.005 & \\
\end{array}$ & $\begin{array}{r}0.009 \\
0.018 \\
\end{array}$ & $\begin{array}{ll} & 0.035 \\
0.021 & \\
\end{array}$ & $\begin{array}{ll} & 0.128 \\
0.043 & \\
\end{array}$ & $0.050 \quad 0.397$ & $\begin{array}{ll} & 0.809 \\
0.077 & \\
\end{array}$ & $0.091 \quad 0.688$ & $0.123 \quad 0.614$ \\
\hline Aluminium & $0.000 \quad 0.000$ & $0.084 \quad 0.092$ & $0.128 \quad 0.150$ & $0.173 \quad 0.658$ & $0.181 \quad 0.703$ & $0.225 \quad 1.228$ & $0.262 \quad 1.179$ & $0.289 \quad 1.129$ \\
\hline Magnesium & $0.000 \quad 0.000$ & $0.000 \quad 0.000$ & $\begin{array}{ll} & 1.038 \\
0.969 & \end{array}$ & $\begin{array}{ll} & 0.679 \\
0.704 & \end{array}$ & $\begin{array}{ll}0.726 & 1.599 \\
\end{array}$ & $\int_{0.947} 1.894$ & $1.115 \quad 1.820$ & ${ }_{1.221} 1.571$ \\
\hline
\end{tabular}

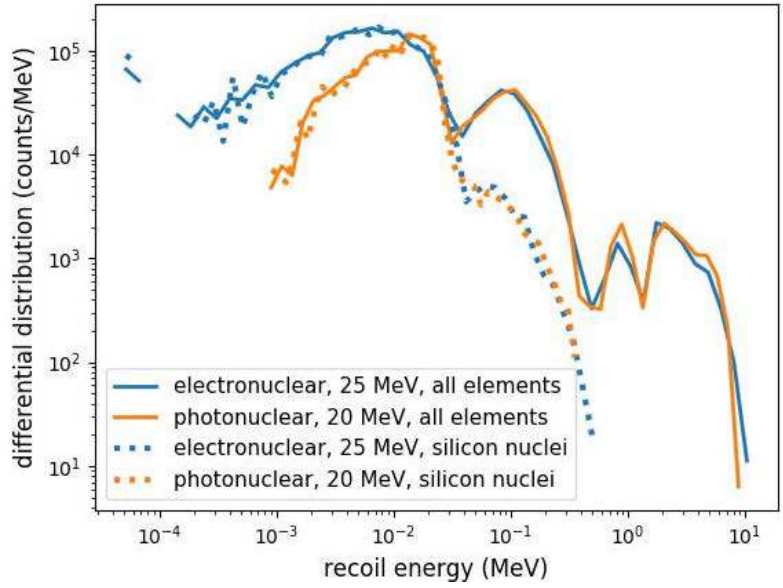

Fig. 7. Recoil energy distribution of secondary generated during nuclear reactions (blue solid line and blue dotted line: all recoil elements and silicon recoil induced by electronuclear reaction; red solid line and red dotted line: all recoil elements and silicon recoil nuclei induced by photonuclear reaction).

where $\sigma_{I}$ is the cross section for the interaction $I, \eta$ is the atomic density of the target material if $I=$ [elastic Coulomb scattering, electronuclear, bremsstrahlung nuclear] or the electron density if $I=$ direct ionization, and $P\left(E_{\mathrm{dep}}>E_{c}\right)$ is the probability of the particle to deposit an energy $E_{\text {dep }}$ higher than a critical energy $E_{c}$.

\section{NumericAl Results}

\section{A. Electro- and Photonuclear Reactions}

In addition to the knowledge of the probability of occurrence of electronuclear and photonuclear reactions, we need to evaluate the energies deposited by secondary populations. Table IV summarizes the important information about recoil energy of secondary elements generated during a nuclear reaction. Two nuclei groups have been distinguished: light

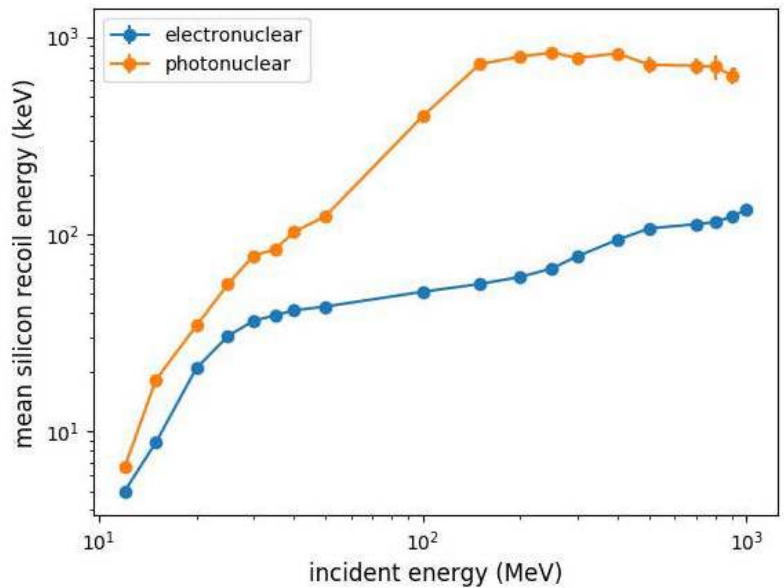

Fig. 8. Mean silicon recoil energy induced by electronuclear and photonuclear reactions as a function of incident energy.

nuclei including elements with atomic number lower than 6 (carbon atom) and heavy nuclei for the others. As mentioned above, electronuclear and photonuclear reactions are closely connected.

Fig. 7 reveals strong similarity between recoil energy distributions of these nuclear reactions. The heaviest recoil elements are likely to deposit the most ionizing energy. In first order, we can focus our analysis on the silicon recoil nuclei. Fig. 8 presents the mean silicon recoil energy. We note that photonuclear reactions produce more energetic recoil elements than electronuclear reactions (as can also be seen in Table IV). This is due to the fact that the incident photon is completely absorbed during the reaction and therefore yields all its energy. This is not the case for incident electrons, which continue to exist after the interaction, and so they cannot deposit all their energy.

If we consider the energy range [10-100] MeV, electronuclear and photonuclear reactions produce recoil silicon with 
TABLE V

CONVERSION BETWEEN TOTAL DEPOSITED ENERGY AND IONIZING Deposited Energy for Some MeAn SillCon Recoil Energies INDUCED B Y ELECTRONUCLEAR AND PHOTONUCLEAR REACTIONS

\begin{tabular}{|c|c|c|c|c|}
\hline Energy $(\mathrm{MeV})$ & 12 & 15 & 20 & 100 \\
\hline$<E_{S i}>_{e^{-}}(\mathrm{keV})$ & 5 & 9 & 21 & 51 \\
\hline Ionizing energy $(\mathrm{keV})$ & 3.6 & 6.3 & 13.5 & 28.9 \\
\hline$<E_{S i}>_{\gamma}(\mathrm{keV})$ & 6.5 & 18.25 & 34.5 & 398 \\
\hline Ionizing energy $(\mathrm{keV})$ & 4.6 & 11.9 & 20.8 & 114.7 \\
\hline
\end{tabular}

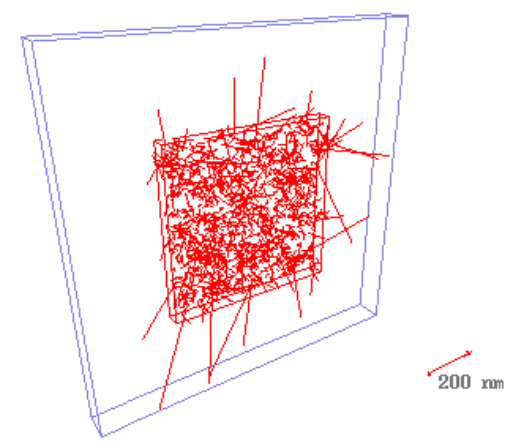

Fig. 9. Geometry used to estimate secondary electrons deposited energy.

on average energy of 50 and $400 \mathrm{keV}$, respectively. This corresponds to a projected range (in silicon material) of about 70 and $535 \mathrm{~nm}$, respectively [27]. In the rest of this paper, we assume that recoil elements will deposit all their energies in the sensitive volume. Therefore, we overestimate electronuclear and photonuclear contributions in our calculations of SEU rates, but this will not change our conclusions. However, we take into account the Lindhard partition function [28] to evaluate the ionizing part of total deposited energy (Table V).

For critical energies of some kiloelectronvolts (corresponding to the sensitivity of current memory devices [3]), electronuclear and photonuclear reactions induce systematically SEUs for incident energy above $20 \mathrm{MeV}$.

\section{B. Deposited Energy by Secondary Electrons}

In addition to the probability to create a secondary, we must realize the transport of these particles. Indeed, treat the secondary by considering only the continuous energy loss is not relevant given the dimensions involved. We have developed a specific application to address this issue. We have performed a parametric study by randomly placing electrons with also random directions (isotropic) in different volume sizes. Our objective was to evaluate the probability of an electron with an energy $T$ to deposit an energy higher than a certain critical energy. Fig. 9 shows a silicon sensitive volume of $50 \times$ $600 \times 600 \mathrm{~nm}^{3}$ randomly bombarded from the inside by 1-keV electrons which, roughly, remained trapped inside (they deposit all their energy), using the MicroElec module [22].

This application allows us to evaluate $P\left(E_{\mathrm{dep}}>E_{c}\right)$ in (3). Indeed, we cannot simply consider the stopping power

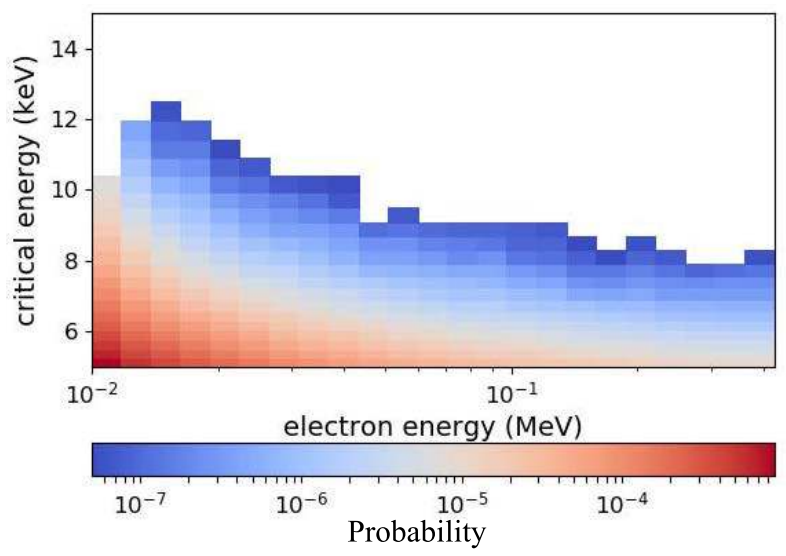

Fig. 10. Probability to reach critical energy as a function of initial secondary electron energies in silicon $300 \times 300 \times 250 \mathrm{~nm}^{3}$ sensitive volume (color in $\log$ scale).

(continuous energy loss). These are relatively rare events that can lead to sufficient deposited energy to trigger upsets. Fig. 10 presents this probability for $10-500-\mathrm{keV}$ electrons in a silicon sensitive volume of $300 \times 300 \times 250 \mathrm{~nm}^{3}$. This study was done for a wide energy range of electrons (kiloelectronvolts up to megaelectronvolts) and some volume sizes (10 up to $500 \mathrm{~nm}$ for depth and $10 \mathrm{~nm}$ up to $1 \mu \mathrm{m}$ for lateral sizes). The higher the initial electron energy, the lower the energy it deposits. In the case of $300 \times 300 \times 250 \mathrm{~nm}^{3}$, regardless of initial electron energy, it is very difficult to deposit more than $\sim 10 \mathrm{keV}$ (Fig. 10).

Thus, the calculations of direct ionization contribution are done in two steps which allows us to better understand what happens, and it is a way to get good statistics in relatively reasonable CPU time: first, the energy distribution is calculated analytically for a given incident electron energy (using dielectric theory), then this distribution is weighted by the simulations of energy deposition in a given volume. We can do that because the ranges of high-energy electrons are largely greater than thicknesses used during our simulations.

\section{COMPARISON With EXPERIMENTAL MEASUREMENTS}

\section{A. Experimental Data}

There are some experimental measurements of electroninduced SEE cross section in [2], [5], [29], and [30]. Our calculations have been compared to SEU cross sections measured on the 45-nm FPGA from Xilinx Spartan 6 [2], [5]. (XC6SLX9 version has been used.) This device has been irradiated during previous campaigns with various energies going from 10 up to $20 \mathrm{MeV}$. The memory tested during the irradiation is the block RAM. That device has been tested at ONERA by means of an electron accelerator Van de Graaff, with a lower energy (1 MeV) than those tested by Samaras et al. [2] and Gadlage et al. [5]. The same configurations were applied: to increase the SEU sensitivity, internal power supply was reduced ( $V_{c c}$ core value was set at 0.672 V) by Samaras et al. [2], while Gadlage et al. [5], [29] have kept the nominal voltage $(1.2 \mathrm{~V})$. Therefore, two runs with different supply voltages have been performed. Table VI 


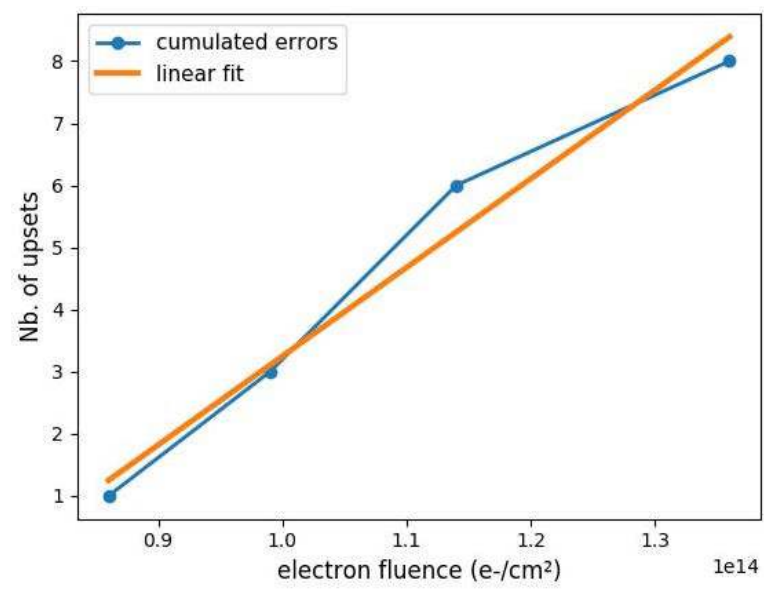

Fig. 11. Accumulated detected upsets as a function of electron fluence during the run, where the reduced voltage was applied. The number of upsets was linear with electron fluence.

TABLE VI

Results Obtained During 1-MeV Electron IRRAdiation on SPARTAN 6 FPGA WITH REDUCED POWER SUPPLY

\begin{tabular}{|c|c|c|c|}
\hline Voltage & Fluence $\left(\mathrm{e}-/ \mathrm{cm}^{2}\right)$ & SEU & SET \\
\hline reduced & $1.5 \cdot 10^{14}$ & 8 & 1 \\
\hline nominal & $1 \cdot 10^{14}$ & 2 & 1 \\
\hline
\end{tabular}

summarizes the obtained results. In addition, the board of the DUT was not designed with actives devices, as was done by Samaras et al. [2].

To discriminate SET to SEU, every bit memory is read twice. The values obtained are compared to a reference memory. At first reading, if the value read is different than the reference value, a second reading is done after a short period of time. If the error persists, we count one SEU else, we count one SET.

The number of measured events corresponds to an SEU cross section of $\sigma_{\text {reduced }} \sim 5.3 \times 10^{-14} \mathrm{~cm}^{2} /$ device and $\sigma_{\text {nominal }} \sim 2 \times 10^{-14} \mathrm{~cm}^{2} /$ device for, respectively, reduced and nominal applied voltages. As expected, reduced voltage leads to an increase in measured upsets. The devices receive a relatively high ionizing dose level (3.675 and 2.45 Mrad), but the SEU cross section is expected to be reliable since a constant upset rate has been measured as a function of the incident fluence as can be seen in Fig. 11 for reduced voltage case (worst dose level). Concerning nominal voltage case, the first SEU has been measured at a fluence level of $3.8 \times 10^{13} \mathrm{e}-/ \mathrm{cm}^{2}$ and the second at $7.1 \times 10^{13} \mathrm{e}-/ \mathrm{cm}^{2}$, which is also consistent. In addition, after irradiation, the devices have remained perfectly functional with the write/read processes totally operational.

We have also considered the possibility that our results were due to photodroop because of photocurrent, but Gadlage et al. [5] had already investigated the issue and had demonstrated that below about $6 \times 10^{10} \mathrm{e}-/ \mathrm{cm}^{2} /$ pulse,

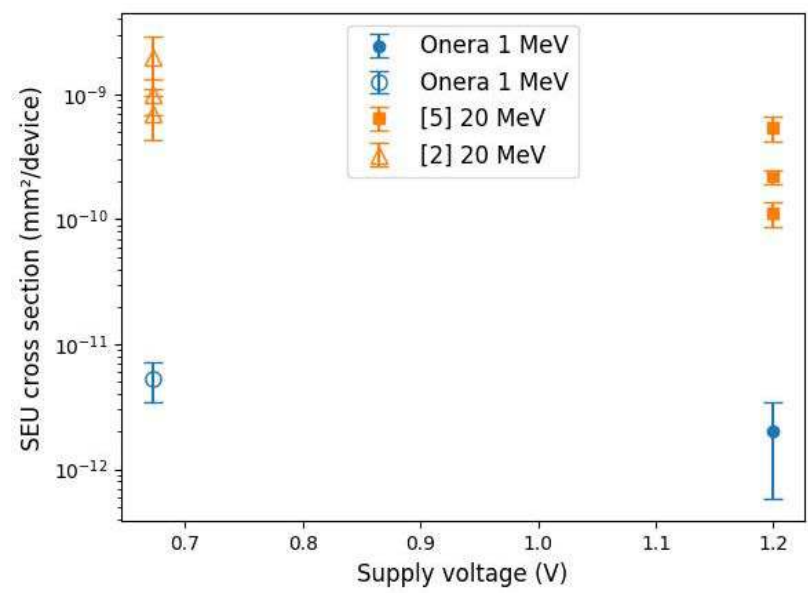

Fig. 12. SEU cross section as a function of supply voltage. Two energies and voltages are compared.

electron fluence does not cause prompt dose-type upset. In the case of the Van de Graaff accelerator used during our tests, the flux applied was about $2.5 \times 10^{10} \mathrm{e}-/ \mathrm{cm}^{2} / \mathrm{s}$, which is largely below the dose rates used by Samaras et al. [2] and Gadlage et al. [5].

It may be interesting to note here the clearly apparent consequence of the reduction of supply voltage (Fig. 12). A factor of 3 and 4 can be noticed depending on the applied supply voltage (0.672 and $1.2 \mathrm{~V})$.

\section{B. Comparisons With Numerical Calculations}

Four cases corresponding to four sensitive volumes are investigated. The calculations have been performed with two sets of parameters proposed by Samaras et al. [2] and Gadlage et al. [5] and intermediate cases have been investigated. The sensitive depths used by Samaras et al. [2] and Gadlage et al. [5] are very different: 50 and $500 \mathrm{~nm}$. The first one is more relevant for SOI architecture. However, as shown in Fig. 13, by adapting the critical charge to match with the experimental data, these different cases lead to the same conclusions.

First of all, the presence of an SEU threshold around $\sim 10 \mathrm{MeV}$ attributed to elastic Coulombic interaction in [3] is confirmed (Fig. 13). The electronuclear process is at least two orders of magnitude lower. Second, the contribution of the Bremsstrahlung is demonstrated to be negligible. Only the major contributions are presented in Fig. 13. Above $10 \mathrm{MeV}$, the direct ionization process is dominated by the Coulomb elastic scattering. But below $10 \mathrm{MeV}$, the former becomes the dominant process. It has to be noted that the ionization process is much more sensitive to critical energy than the Coulomb elastic interaction. The level of sensitivity below $10 \mathrm{MeV}$, for a given sensitive volume, depends closely on the chosen critical energy (much more than elastic Coulombinduced SEUs, as seen in Fig. 13). This means that from device to device we can expect different behaviors presenting or not a threshold around $10 \mathrm{MeV}$ according to the relative level of the direct ionization and Rutherford processes. Some devices having relatively low critical energy could show flat cross section dominated by the direct ionization process, independent of the 

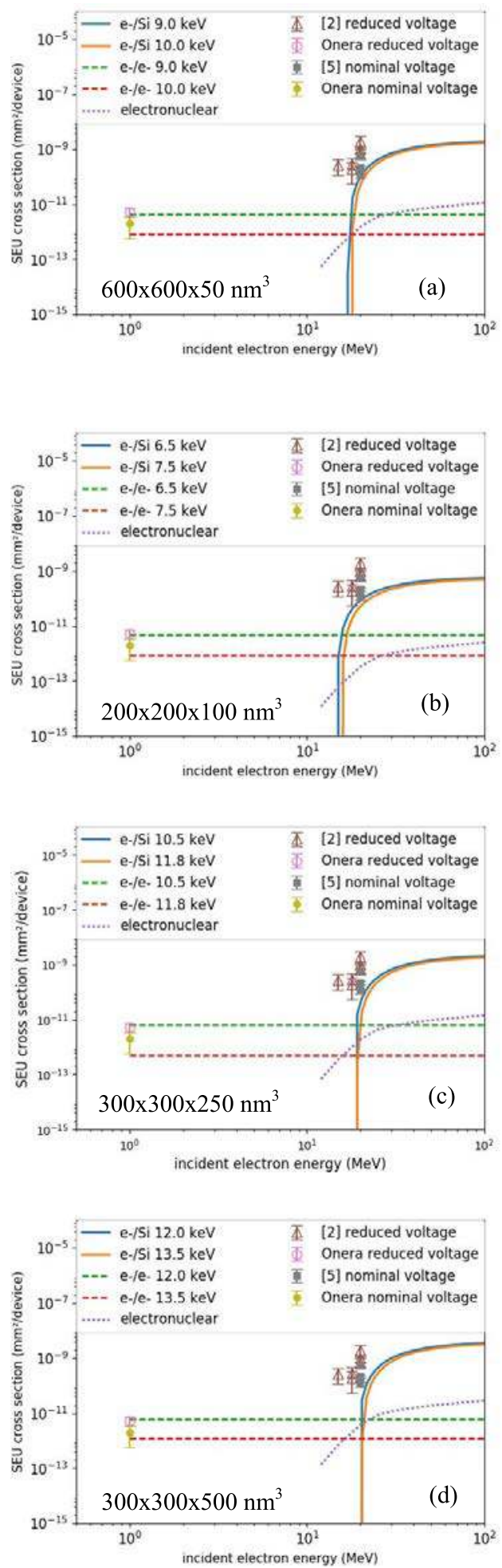

Fig. 13. Electron-induced SEU contributions for different critical energies and sensitive volumes. (a) $600 \times 600 \times 50 \mathrm{~nm}^{3}$ [2], [3], 0.40-0.45 fC. (b) 200 $\times 100 \times 100 \mathrm{~nm}^{3}, 0.29-0.34 \mathrm{fC}$. (c) $300 \times 300 \times 250 \mathrm{~nm}^{3}, 0.47-0.53 \mathrm{fC}$. (d) $300 \times 300 \times 500 \mathrm{~nm}^{3}$ [5], $0.53-0.60 \mathrm{fC}$.

incident energy. The objective of this paper was not to fit the experimental data but to discriminate the most important processes. As seen in Fig. 13, the RPP approximation is not relevant to analyze the consequence of voltage variation and underestimates elastic Coulombic SEU cross sections in the case where direct ionization SEU cross sections match very well with the experimental data.

\section{CONCLUSION}

Among all the physical mechanisms studied in this paper, two main processes are identified to be at the origin of the observed SEUs. The Rutherford electron/nuclei elastic scattering, which is demonstrated to be the dominant process above $\sim 10 \mathrm{MeV}$ and the inelastic electron/electron scattering which is the most important process below $\sim 10 \mathrm{MeV}$. The electronuclear interactions contribute two orders of magnitude less than the Rutherford scattering, and the bremsstrahlung-induced processes remain negligible. The inelastic electron/electron interactions are demonstrated to be the dominant process below $10 \mathrm{MeV}$, but the level of the SEU cross section can vary significantly and is strongly dependent on the critical energy. More detailed simulations taking into account the charge collection mechanisms, based on TCAD and SPICE modeling, could be interesting in order to take into account neighboring structures, transient mechanisms, and complete transportation of secondary nuclei.

\section{REFERENCES}

[1] M. P. King et al., "Electron-induced single-event upsets in static random access memory," IEEE Trans. Nucl. Sci., vol. 60, no. 6, pp. 4122-4129, Dec. 2013.

[2] A. Samaras et al., "Experimental characterization and simulation of electron-induced SEU in 45-nm CMOS technology," IEEE Trans. Nucl. Sci., vol. 61, no. 6, pp. 3055-3060, Dec. 2014.

[3] C. Inguimbert, R. Ecoffet, and D. Falguère, "Electron induced SEUs: Microdosimetry in nanometric volumes," IEEE Trans. Nucl. Sci., vol. 62, no. 6, pp. 2846-2852, Dec. 2015.

[4] J. M. Trippe et al., "Electron-induced single event upsets in $28 \mathrm{~nm}$ and $45 \mathrm{~nm}$ bulk SRAMs," IEEE Trans. Nucl. Sci., vol. 62, no. 6 , pp. 2709-2716, Dec. 2015.

[5] M. J. Gadlage, A. H. Roach, A. R. Duncan, M. W. Savage, and M. J. Kay, "Electron-induced single-event upsets in 45-nm and 28-nm bulk CMOS SRAM-based FPGAs operating at nominal voltage," IEEE Trans. Nucl. Sci., vol. 62, no. 6, pp. 2717-2724, Dec. 2015.

[6] C. Inguimbert, P. Caron, R. Ecoffet, D. Falguère, J. F. Roussel, and S. Duzellier, "Electron induced SEUs in latest technologies," presented at the Int. Astron. Congr., Mexico, 2016.

[7] A. Akkerman, J. Barak, and N. M. Yitzhak, "Role of elastic scattering of protons, muons, and electrons in inducing single-event upsets," IEEE Trans. Nucl. Sci., vol. 64, no. 10, pp. 2648-2660, Oct. 2017.

[8] J. Allison et al., "Geant4 developments and applications," IEEE Trans. Nucl. Sci., vol. 53, no. 1, pp. 270-278, Feb. 2006.

[9] S. Agostinelli, "GEANT4-a simulation toolkit," Nucl. Instrum. Methods Phys. Res. A, Accel. Spectrom. Detect. Assoc. Equip., vol. 506, no. 3, pp. 250-303, 2003.

[10] Geant4 Collaboration. Geant4 10.2 Geant4 Physics Reference Manual Version 10.2. Accessed: Sep. 23, 2017. [Online]. Available: http://www.geant4.org/

[11] M. J. Berger, J. S. Coursey, M. A. Zucker, and J. Chang. ESTAR, PSTAR, and ASTAR: Computer Programs for Calculating Stopping-Power and Range Tables for Electrons, Protons, and Helium Ions. National Institute of Standards and Technology, Gaithersburg, MD, USA. Accessed: Sep. 23, 2017. [Online]. Available: http://physics.nist.gov/Star

[12] M. J. Boschini et al. (Dec. 2011). "Nuclear and non-ionizing energyloss of electrons with low and relativistic energies in materials and space environment." [Online]. Available: https://arxiv.org/abs/1111.4042

[13] J. Ahrens et al., "Total nuclear photon absorption cross sections for some light elements," Nucl. Phys. A, vol. 251, no. 3, pp. 479-492, Oct. 1975.

[14] J. M. Wyckoff, B. Ziegler, H. W. Koch, and R. Uhlig, "Total photonuclear cross sections for low atomic number elements," Phys. Rev., vol. 137, no. 3B, pp. B576-B594, Feb. 1965. 
[15] M. V. Kossov, "Approximation of photonuclear interaction crosssections," Eur. Phys. J.-Hadrons Nucl., vol. 14, no. 3, pp. 377-392, Jul. 2002.

[16] J. P. Wellisch, M. Kossov, and P. Degtyarenko. (Jun. 2003). "Electro and gamma nuclear physics in Geant4." [Online]. Available: https://arxiv.org/ abs/nucl-th/0306012

[17] A. Heikkinen, N. Stepanov, and J. P. Wellisch. (Jun. 2003). "Bertini intra-nuclear cascade implementation in Geant4." [Online]. Available: https://arxiv.org/abs/nucl-th/0306008

[18] V. Uzhinsky, "Development of the Fritiof Model in Geant4," in Proc. Joint Int. Conf. Supercomput. Nucl. Appl. Monte Carlo $(\mathrm{SNA}+\mathrm{MC})$, Tokyo, Japan, 2010, pp. 1-4.

[19] S. M. Seltzer and M. J. Berger, "Bremsstrahlung energy spectra from electrons with kinetic energy $1 \mathrm{keV}-10 \mathrm{GeV}$ incident on screened nuclei and orbital electrons of neutral atoms with $\mathrm{Z}=1-100$," Atomic Data Nucl. Data Tables, vol. 35, no. 3, pp. 345-418, Nov. 1986.

[20] M. J. Berger et al. Accessed: Sep. 23, 2017. XCOM: Photon Cross Sections Database. [Online]. Available: https://physics.nist.gov/

[21] M. Tali et al., "High-energy electron-induced SEUs and Jovian environment impact," IEEE Trans. Nucl. Sci., vol. 64, no. 8, pp. 2016-2022, Aug. 2017.

[22] A. Valentin, M. Raine, J.-E. Sauvestre, M. Gaillardin, and P. Paillet, "Geant4 physics processes for microdosimetry simulation: Very low energy electromagnetic models for electrons in silicon," Nucl. Instrum. Methods Phys. Res. B, Beam Interact. Mater. At., vol. 288, pp. 66-73, Oct. 2012
[23] C. Inguimbert and S. Duzellier, "SEU rate calculation with GEANT4 (comparison with CREME 86)," in Proc. 7th Eur. Conf. Radiat. Effects Compon. Syst. (RADECS), 2003, pp. 243-248.

[24] J. C. Pickel and J. T. Blandford, "Cosmic-ray-induced errors in MOS devices," IEEE Trans. Nucl. Sci., vol. NS-27, no. 2, pp. 1006-1015, Apr. 1980.

[25] E. Petersen, Single Event Effects in Aerospace. New York, NY, USA: Wiley, 2011.

[26] R. C. Alig and S. Bloom, "Electron-hole-pair creation energies in semiconductors," Phys. Rev. Lett., vol. 35, pp. 1522-1525, Dec. 1975.

[27] J. F. Ziegler, M. D. Ziegler, and J. P. Biersack, "SRIM-The stopping and range of ions in matter (2010)," Nucl. Instrum. Methods Phys. Res. B, Beam Interact. Mater. At., vol. 268, nos. 11-12, pp. 1818-1823, Jun. 2010.

[28] I. Jun, "Effects of secondary particles on the total dose and the displacement damage in space proton environments," IEEE Trans. Nucl. Sci., vol. 48, no. 1, pp. 162-175, Feb. 2001.

[29] M. J. Gadlage, A. H. Roach, A. R. Duncan, A. M. Williams, D. P. Bossev, and M. J. Kay, "Soft errors induced by high-energy electrons," IEEE Trans. Device Mater. Rel., vol. 17, no. 1, pp. 157-162, Mar. 2017.

[30] M. J. Gadlage, A. H. Roach, A. R. Duncan, A. M. Williams, D. P. Bossev, and M. J. Kay, "Multiple-cell upsets induced by single high-energy electrons," IEEE Trans. Nucl. Sci., vol. 65, no. 1, pp. 211-216, Jan. 2018. 